\title{
BMJ Open Birth experience during COVID-19 confinement (CONFINE): protocol for a multicentre prospective study
}

\author{
Charline Bertholdt (D) , ${ }^{1,2}$ Jonathan Epstein, ${ }^{3}$ Claire Banasiak, ${ }^{4}$ Fabienne Ligier, ${ }^{5}$ \\ Sandra Dahlhoff, ${ }^{6}$ Marie France Olieric, ${ }^{7}$ Nicolas Mottet, ${ }^{8}$ Marine Beaumont, ${ }^{4}$ \\ Olivier Morel ${ }^{1,2}$
}

To cite: Bertholdt C, Epstein J, Banasiak C, et al. Birth experience during COVID-19 confinement (CONFINE): protocol for a multicentre prospective study. BMJ Open 2020;10:e043057. doi:10.1136/ bmjopen-2020-043057

- Prepublication history and additional material for this paper are available online. To view these files, please visit the journal online (http://dx.doi. org/10.1136/bmjopen-2020043057).

Received 24 July 2020 Revised 05 0ctober 2020 Accepted 18 November 2020

Check for updates

(c) Author(s) (or their employer(s)) 2020. Re-use permitted under CC BY-NC. No commercial re-use. See rights and permissions. Published by BMJ.

For numbered affiliations see end of article.

Correspondence to Dr Charline Bertholdt; charline.bertholdt@gmail.com

\section{ABSTRACT}

Introduction The absence of companionship during childbirth is known to be responsible for negative emotional birth experience, which can increase the risk of postpartum depression and post-traumatic stress disorder. The context of COVID-19 epidemic and the related confinement could increase the rate of negative experience and mental disorders. The main objective is to compare, in immediate post partum, the maternal sense of control during childbirth between a group of women who gave birth during confinement ('confinement' group) versus a group of women who gave birth after confinement but in the context of epidemic ('epidemic' group) versus a group of control women ('control' group; excluding confinement and epidemic context).

Methods and analysis This is a national multicentre prospective cohort study conducted in four French maternity units. We expect to include 927 women in a period of 16 months. Women will be recruited immediately in post partum during three different periods constituting the three groups: 'confinement'; 'epidemic' and 'control' group. The maternal sense of control will be evaluated by the Labour Agentry Scale questionnaire completed immediately in post partum. Postnatal depression (Edinburgh Postnatal Depression Scale), post-traumatic stress disorder (Impact of Event Scale-Revised) and breast feeding (evaluative statement) will be evaluated at 2 months post partum.

Ethics and dissemination The study was approved by the French Ethics Committee, the CPP (Comité de Protection des Personnes) SUD OUEST ET OUTRE-MER IV on 16th of April 2020 with reference number CPP202004-040. The results of this study will be published in a peer-reviewed journal and will be presented at relevant conferences.

Trial registration number NCT04348929.

\section{INTRODUCTION}

In the management of a pandemic, the respect for social distancing is very important to reduce infections. COVID-19 epidemic, due to SARS-CoV-2, has forced many countries around the world to impose a confinement period in order to obtain efficient social distancing.
Strengths and limitations of this study

This is a prospective controlled study with a homogeneous population.

- The study is planned at different times of the COVID-19 epidemic.

- The study is designed with questionnaires with international validation.

- There is a risk of lost to follow-up for secondary objectives.

- The evaluation of mother-child assessment is not planned.

These measures led to a reorganisation of health units. In obstetrics, the delivery should be conducted, at least, with surgical mask for both woman and caregiver, face shield and surgical gown for caregiver only. ${ }^{12}$ Several societies, especially in Europe, recommended to limit the number of accompanying persons during childbirth and post partum, which can be particularly stressful in association with all other protective measures and the context of epidemic. $^{2}$

In France, confinement, started at March 17 , conducted all maternity to reorganise and limit contact. ${ }^{3}{ }^{4}$ When usually a companionship is almost permanently authorised in the delivery room and when the visits of friends or family are possible in postnatal period, to support the woman, the maternities have severely limited or even prohibited the presence of the companion during childbirth and prohibited all visits in postnatal hospitalisation period. Moreover, isolation may continue on home return if it occurs during confinement.

In the literature, the absence of companionship during childbirth has been associated with a negative emotional birth experience. ${ }^{5}$ This experience may be responsible for an increased risk of postpartum depression (PPD) and post-traumatic stress disorder 
(PTSD) ${ }^{5-10}$ In the longer term, this increase in mental disorders has a negative impact on the mother-child interaction but also on the marital and family relationships. ${ }^{11}$ In addition, the rate of postpartum maternal suicide is increased in the event of mental disorders. ${ }^{12-14}$

However, these data cannot be extrapolated to the current context where the absence of companionship is imposed, and not as a result of a choice or a custom as found in the literature. Moreover, the impact of close relation absence in immediate post partum, that is, during the hospitalisation in maternity, has never been evaluated since this situation has not occurred in decades.

While women are already consider at risk of mental health disorders during the postpartum period, $10 \%$ for PPD and 3\% for PTSD, this is not integrated yet into current support. ${ }^{67}$ In this unpredictable situation where the woman finds herself alone, the negative impact of birth experience is potentially increased, as the rate of mental disorders.

It is a very important topic to consider the psychological impact of confinement in this fragile population. ${ }^{15-19}$ The implementation of these restrictions in maternity units is sometimes associated to a real obstetric violence by women's associations. In this new era where everything is done and thought to improve the well-being of women during this period, it seems essential to assess the impact of this unprecedented, and probably not unique, situation.

\section{METHODS AND ANALYSIS Objectives}

The primary objective is to compare, in immediate post partum, the maternal sense of control during childbirth between a group of women who gave birth during confinement ('confinement' group) versus a group of women who gave birth after confinement but in the context of epidemic ('epidemic' group) versus a group of control women ('control' group; excluding both confinement and epidemic context).

The secondary objectives are:

1. To compare, at 2 months post partum, the maternal sense of control during childbirth between the three groups.

2. To compare, at 2 months post partum, the scoring of self-questionnaire Edinburg Postnatal Depression Scale (EPDS) for the postnatal depression between the three groups.

3. To compare, at 2 months post partum, the scoring of self-questionnaire Impact of Event Scale-Revised (IES-R) for PTSD, between the three groups.

4. To compare, at 2 months post partum, the breast feeding between the three groups.

5. To compare, at 2 months post partum, the quality of life between the three groups.

6 . To compare the evolution of quality of life at 2 months of post partum between the three groups.
7. To compare the rate of postnatal depression between the three groups.

\section{Trial design}

The CONFINE protocol is a national, multicentre prospective cohort study concerning four French centres: Centre Hospitalier Régional Universitaire (CHRU) of Nancy, Centre Hospitalier Universitaire of Besançon and Centre Hospitalier Régional Metz-Thionville which include two hospitals: Hôpital Bel-Air and Hôpital mèreenfant Mercy.

\section{Study population}

Women will be informed and recruited after birth, in immediate post partum, over a period of 16 months for the three groups. The inclusion and exclusion criteria are reported in table 1 . The study information and consent collection will be provided by the investigators (obstetricians or midwives) (see online supplemental file 1). Women selection will be done on consecutive cases and depending on the availability of the investigators.

\section{Outcomes}

The primary endpoint is the measure of self-questionnaire Labour Agentry Scale (LAS) ${ }^{20}$ in immediate post partum (defined as the duration of hospital stay, approximately 4 days).

The secondary endpoints are:

1. Measure of self-questionnaire LAS at 2 months post partum. ${ }^{20}$

2. Measure of self-questionnaire EPDS at 2 months post partum. ${ }^{21}$

3. Measure of self-questionnaire IES-R at 2 months post partum. ${ }^{22}$

4. Breastfeeding statement at 2 months post partum.

5. Measure of self-questionnaire 12-Item Short Form Health Survey (SF-12) quality of life at 2 months post partum. $^{23}$

6. Measure of self-questionnaire SF-12 quality of life in immediate and at 2 months post partum.

7. Diagnosis of postnatal depression (made by a specialist).

\section{Participant timeline}

The enrolment of women started in April 2020. The recruitment should be achieved by August 2021. The flow chart of patient participation is presented in figure 1.

\section{Premature ending of patient participation}

Each person can stop participating in the research at any time and for whatever reason.

The investigator may temporarily or permanently interrupt a person's participation in the research for any reason that has an impact on her safety or that would best serve the interests of the person who is suitable for research.

In the event of a premature ending or in the event of consent withdrawal, the withdrawal shall not affect the activities carried out and the use of data obtained on the 
Table 1 Inclusion and exclusion criteria

\begin{tabular}{|c|c|}
\hline Inclusion criteria & Exclusion criteria \\
\hline $\begin{array}{l}\text { Women having just given birth from } 37 \text { weeks of gestation (singleton } \\
\text { pregnancy) }\end{array}$ & Newborn with congenital abnormalities \\
\hline Woman over 18 years old & $\begin{array}{l}\text { Woman with psychiatric disorders such as depressive } \\
\text { syndrome }\end{array}$ \\
\hline $\begin{array}{l}\text { Woman having received complete information on the organisation of } \\
\text { the research and having given her informed consent in written form }\end{array}$ & $\begin{array}{l}\text { Major mentioned in articles L.1121-6 and L.1121-8 of } \\
\text { Health Public Code }\end{array}$ \\
\hline \multicolumn{2}{|l|}{ Specific inclusion criteria according to the group } \\
\hline \multicolumn{2}{|l|}{$\begin{array}{l}\text { For 'confinement' group: delivery during COVID-19 confinement } \\
\text { period }\end{array}$} \\
\hline $\begin{array}{l}\text { For 'control' group: start of pregnancy after confinement and delivery } \\
\text { after the withdrawal of all sanitary measures (mask, social distancing, } \\
\text { limited visits during postpartum immediate) }\end{array}$ & \\
\hline
\end{tabular}

basis of informed consent before it has been withdrawn, unless the person indicates in writing that she objects to their use.

When a patient withdraws her consent, the data acquired before the withdrawal will be available for the statistical analysis unless refusal from the patient.

\section{Follow-up}

No specific follow-up is organised for patients ending, prematurely or not, their participation in the study. Pregnancy course and outcome of all participants will be recorded on electronic case report forms (eCRFs) and added to the database. If the responses to the questionnaires suggest a mental disorder, the woman will quickly benefit (within a week) of an appointment with a psychiatrist in order to confirm, or not, the presence of a pathology and thus suggest the most appropriate care. Woman can also, in accordance with current healthcare practices, request to benefit from psychological support regardless of the results of the questionnaires.

\section{Sample size consideration}

According to the literature, the LAS has an SD of 20, and has no known clinically important difference. To show a

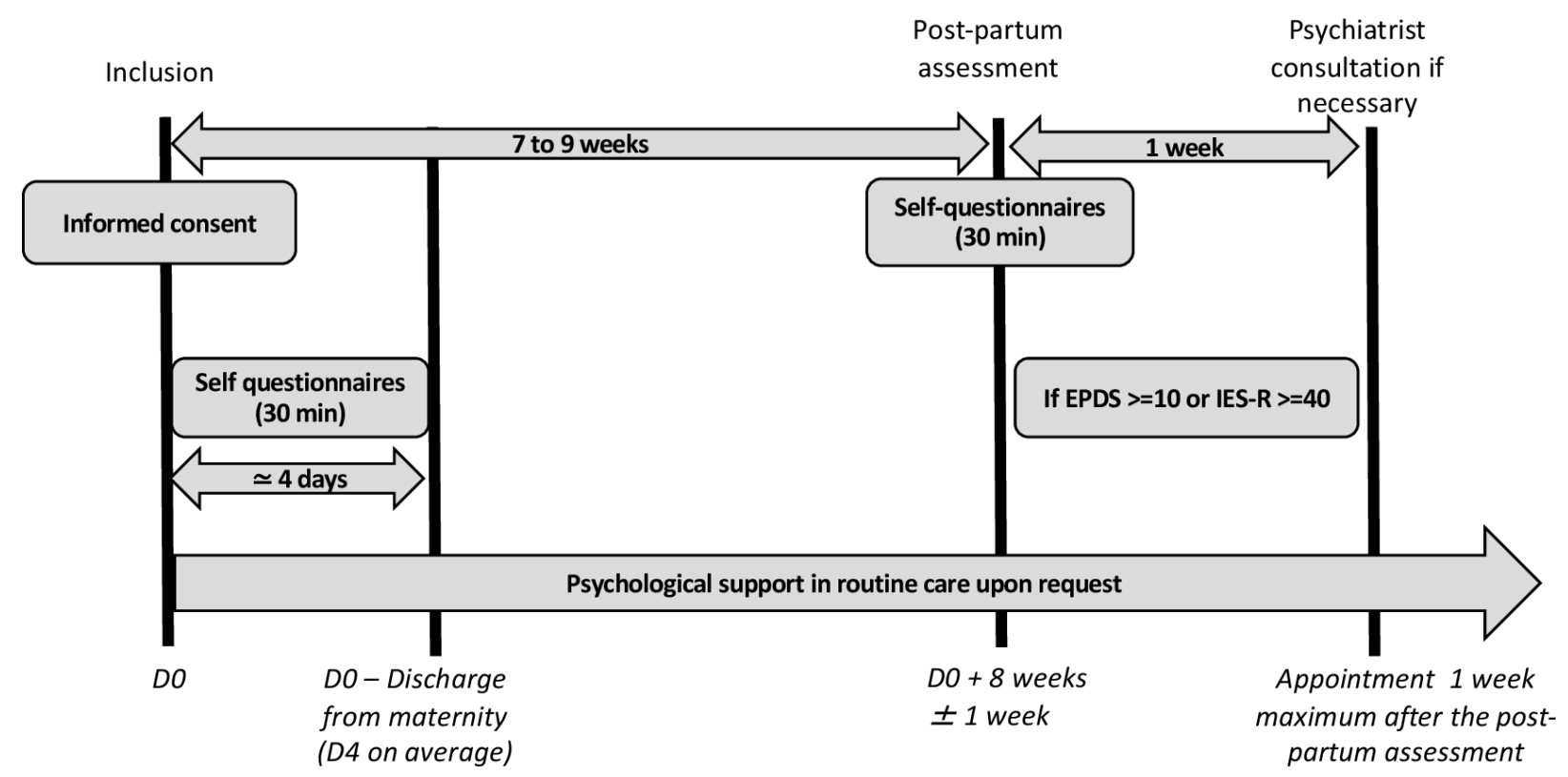

Figure 1 Participant timeline. EPDS, Edinburgh Postnatal Depression Scale; IES-R, Impact of Event Scale-Revised. 
standardised difference of 0.25 (small to medium effect) between the 'confinement' group and the two other groups, for a power of $80 \%$ and a false discovery rate for multiple comparisons between the groups at $5 \%$, and because the duration of confinement was uncertain, the number of subjects to be included in the confinement group was 200-300 using a scalable approach to ensure that a minimum acceptable power was achieved while optimising the statistical power of the study, with adjustment of the number of subjects in the other groups.

Once the final number of women included in the 'confinement' group was set, an analysis of variance simulation found that 310 women were needed in the other two groups, which was increased by more than $5 \%$ to 330 in order to have a margin of error. The calculated total necessary sample size was 927 women (267 in the 'confinement' group and 330 in each of the other two groups). As this is an observational study, groups will not be perfectly homogeneous, however the known maternal characteristics will be taken into account into the multivariable analysis thus neutralising their effects.

The sample size is designed in order to answer the first objective, which does not use any follow-up data from the women, so the lost to follow-up is not taken into account for the calculation. However, the calculated sample size takes into account the possibility of missing answers at inclusion and was increased accordingly.

\section{Data collection and management}

The collection of women characteristics and immediate postpartum questionnaires will be performed at the time of inclusion in paper version. The postpartum evaluation at 2 months will be performed 8 weeks post partum $( \pm 1$ week) with an online questionnaire. Data for this study will be recorded on an eCRF and are presented in table 2.
The data from all women will be centrally stored in Nancy. Data management will be carried out by Nancy CIC-EC (INSERM CIC 1433). The conditions for data transfer of all or part of the study database are decided by the study sponsor and are the subject of a written contract. This study and the data collected fall within the scope of Reference Methodology MR001.

\section{Statistical analysis}

Variables will be described according to their nature by their frequencies, proportions, means (SDs) and medians (IQR).

Bivariate analyses will be conducted with the appropriate statistical tests, under appropriate assumptions for the test.

Analysis will be carried out in two stages:

- The first stage will take place at the end of the follow-up of the second group, with the analyses described below.

- The second stage will take place at the end of the follow-up of the third group.

In order to avoid inflation of the first-species risk and to keep the total false discovery rate below $5 \%$, the significance threshold for the main analysis of the first stage is set at 0.03 , based on the result of simulations using the hypotheses of expected differences in LAS between groups.

The main analysis will consist of a multivariable linear regression with the LAS score as the dependent variable, the presence of confinement as the explanatory variable and with the variables already known in the literature as co-variables (mode of delivery (spontaneous/intervention), mode of entry into labour (spontaneous/triggered), marital status (married/partner/couple/single) as well as the assumed confounding factors:

Table 2 Data collected

\begin{tabular}{|c|c|}
\hline Maternal characteristic & Parity; conception mode \\
\hline Neonatal outcomes & $\begin{array}{l}\text { Neonatal weight; Apgar score } \\
\text { Umbilical artery } \mathrm{pH} \text {; admission in neonatal intensive care unit }\end{array}$ \\
\hline Questionnaires & $\begin{array}{l}\text { Labour Agentry Scale } \\
\text { Quality of Prenatal Care Questionnaire } \\
\text { Quality of Life 12-item Short Form Health Survey } \\
\text { Edinburg Postnatal Depression Scale } \\
\text { Impact of Event Scale-Revised } \\
\text { Breast feeding }\end{array}$ \\
\hline
\end{tabular}


- Data concerning the woman such as: age, parity, intake of toxic substances (tobacco, alcohol, other toxic substances), COVID-19 status during pregnancy, psychological support/accompaniment accepted or requested by the woman on leaving the maternity hospital, duration of post-delivery confinement.

- Socioeconomic data (number of people in the household, living space, socioprofessional category, financial impact related to COVID-19, level of education).

- Pregnancy and childbirth data such as: presence of the co-parent during the first 8 months of pregnancy, quality of the person accompanying the woman during delivery, time of presence, feeling of the co-parent declared by the woman, use of digital media to compensate for absence, gestational age, mode of conception, pregnancy considered at risk, number of consultations during pregnancy, consultation modality (virtual/on-site) during confinement, hospitalisation during pregnancy, postpartum haemorrhage, perineal injury, other complications of delivery, perceived quality of care (as assessed by the Quality of Prenatal Care Questionnaire selfadministered questionnaire).

- Breastfeeding statement: choice of breast feeding, duration of breast feeding, maternity support assessment to help for breast feeding.

- Child data such as birth weight, Apgar, neonatal hospitalisation.

These confounding factors will be included in the multivariable model if they are associated with the LAS score in the bivariate analyses at a threshold of 0.1.

Analyses for secondary objectives will be conducted on the same principle.

The change between the two measurement times is calculated at the individual level by the difference in score between the two times.

All women participating in the study will be included in the analyses, in case of missing data for multivariable analyses, if data are missing at random the analysis will be done with a listwise deletion, and a sensitivity analysis will be conducted using a multiple imputation method.

\section{Patient and public involvement}

Patients and public were not involved.

\section{Ethics and dissemination}

The study was approved by the French Ethics Committee, the CPP (Comité de Protection des Personnes) SUD OUEST ET OUTRE-MER IV on 16th of April 2020 with reference number CPP2020-04-040. Results of this study will be published in a peer-reviewed journal and will be presented at relevant conferences.

\section{Quality control}

Right of access to data and source documents

The CHRU is responsible for obtaining the agreement of all parties involved in the study so as to guarantee direct access to all study sites, source data, source documents and reports so that the sponsor can control data quality and perform audit.

Investigators will make available the documents and individual data strictly required for monitoring, quality control and audit of the biomedical study to persons having access to these, in accordance with the statutory and regulatory provisions in place (articles L.1121-3 and R.5121-13 of the French Public Health Code).

Any original document or object that allows the existence or accuracy of a data point or information recorded during the study to be proved is defined as a source document.

In accordance with the statutory provisions in place (articles L.1121-3 and R.5121-13 of the French Public Health Code) the persons having direct access to source data will take every precaution required to ensure the confidentiality of information relating to investigational medicinal products, studies, participants, notably concerning the identity of these, as well as the results obtained. These persons, as the investigators themselves, are subject to professional confidentiality.

During the study, or at its conclusion, data collected regarding participants that are sent to the sponsor by the investigators (or all other specialists involved) will be coded by the inclusion number of the patient in the study. At no point should the names of participants or their addresses appear unencrypted. The presentation of the data processing results cannot in any case allow the direct or indirect identification of persons lending themselves to research.

The sponsor will ensure that each study participant has given her written consent for access to her personal data that is strictly required for study quality control.

The data may be transmitted to the companies in the group to which the promoter belongs and to its contractual partners in a form which should not permit the direct or indirect identification of persons lending themselves to research.

\section{Study monitoring}

Monitoring visits (implementation, follow-up and closure) will be performed by the promoting cell of Nancy Délégation à la Recherche Clinique et à l'Innovation-CHRU Nancy.

Depending on monitoring reports and deviations observed, the promoter reserves the right to modify the level of monitoring initially planned.

\section{Potential risks related to the study}

There are no risks for the patient and for the newborn.

\section{Ethical permission}

The sponsor and investigators undertake to carry out this research in accordance with the recommendations of the Helsinki Declaration and its revisions, the European Regulation (EU) No 536/2014 from the European Parliament about clinical trials of medicines for human use, repealing European Directive 2001/20/CE, the No 
2004-806 law of 9 August 2004 about public health policy, the No 2004-800 law of 6 August 2004 about bioethics, the Regulation (EU) 2016/679 of 27 April 2016 on the protection of individuals with regard to the processing of personal data and on the free movement of such data (General Regulation on Data Protection), the No 78-17 law of 6 January 1978 relating to data processing, files and freedoms, the No 2012-300 law of 5 March 2012 about research involving the human person, the 2016-41 law of 26 January 2016 of modernisation of our healthcare system and 2016-800 ordinance of 16 June 2016 relating to researches involving the human person and their implementing decrees.

They undertake to comply with all laws and regulations that may apply to research.

The investigators undertake to respect the protocol in all respects especially with regard to obtaining consent and the notification and follow-up of serious adverse events.

\section{Protocol amendment}

Requests of authorisation and/or opinion about substantial amendments will be addressed by sponsor to regulatory institutions.

By signing this protocol, the investigator commits to submit to the Direction of Clinical Research and Innovation the substantial amendment project and wait for authorisation and/or opinion of regulatory institutions prior to the application of amendment.

\section{Final research report}

The final report of the research will be written collaboratively by the coordinator and the biostatistician mandated for this research. This report will be submitted to each of the investigators for review. Once a consensus has been reached, the final version must be endorsed with the signature of each of the investigators and sent to the sponsor as early as possible after the effective end of the research. A report prepared according to the reference plan of the competent authority must be forwarded to the competent authority and the CPP within a year after the end of the research, understood as being the last follow-up visit of last enrolled subject. This period is abrogated to 90 days in case of premature termination of the research.

\section{DISCUSSION}

The main objective of this study is to evaluate the birth experience, assessed by the maternal sense of control during childbirth, during COVID-19 epidemic and related confinement compared with control groups.

It has already been shown that the current situation is responsible for very significant anxiety, reduced quality of life and maternal depression in pregnant women. ${ }^{24-26}$

To our knowledge, this is the first study evaluating the psychological impact in both immediate and short-term postpartum periods and in comparison with control groups.
All the questionnaires chosen for the study were validated in French or Canadian language. The LAS was chosen for the evaluation of immediate psychological impact because it allows to measure the maternal sense of control during labour and delivery and we think that COVID-19 epidemic, and especially the associated confinement, can upset this feeling. ${ }^{502728}$ Also, we know that a negative feeling of control is known to be associated with postnatal depression and PTSD. ${ }^{6} 72029$

The EPDS was chosen since it is already widely used in the literature for postnatal depression scoring. ${ }^{1421}$ For the evaluation of PTSD, we opted for auto-questionnaires, due to the COVID-19 epidemic and associated confinement. The IES-R, compared with Clinician Administered PTSD Scale, which corresponds to a structured interview during 30 min with a professional, seemed the most suitable regarding our criteria. ${ }^{22}$

There are only two studies in the literature about postnatal depression related to confinement and COVID-19 epidemic. ${ }^{19} 30$ The results from Zanardo's study are interesting and highlight the usefulness of this type of studies. Indeed, they showed a significant increase of EPDS score in COVID-19 group versus control group with a $28 \%$ probability of postnatal depression versus only $12 \%$ in general population. However, the published results were preliminary because the evaluation was made at 2 days post partum only, so it might have reflected baby blues more than real postnatal depression. Moreover, the diagnostic was not confirmed by a psychological specialist, what we will perform in our study.

Two other studies, registered in ClinicalTrials.gov (NCT04368208 and NCT04366817) and evaluating postnatal depression during COVID-19 epidemic, will be started soon but without control group, which is a major difference with the presented protocol.

This proposed study will investigate the potential negative psychological impact of confinement during COVID-19 epidemic. The study design allows us to offer a specific care to women showing distress in order to improve their mental health and avoid serious consequences like failures in mother-infant link, couples' relationship or suicidal ideation. ${ }^{11-14}$

\section{Author affiliations}

${ }^{1}$ Gynecology-Obstetric center, University of Lorraine, CHRU-Nancy, Nancy, France ${ }^{2}$ Inserm, Diagnostic and Interventional Adaptive Imaging, University of Lorraine, Nancy, France

${ }^{3}$ Inserm, CIC Epidémiologie Clinique, CHRU-Nancy, Nancy, France

${ }^{4}$ Inserm, CIC Innovation Technologique, CHRU-Nancy, Nancy, France

${ }^{5}$ PUPEA, Nancy Psychotherapy Center-EA 4360 APEMAC, MICS team, University of Lorraine, Nancy, France

${ }^{6}$ Obstetric Gynecology, CHR Metz-Thionville, Metz Mother and Child Hospital, Peltre, France

${ }^{7}$ Obstetric Gynecology, CHR Metz-Thionville, Bel-Air Hospital, Thionville, France ${ }^{8}$ Obstetric Gynecology, CHU Besançon Jean-Minjoz Hospital, Besancon, France

Contributors $\mathrm{CB}, \mathrm{OM}, \mathrm{JE}, \mathrm{MB}$ and $\mathrm{CB}$ contributed to the conception and design of the study. $O M$ and $\mathrm{CB}$ are the coordinating investigators. $\mathrm{CB}$ is the study project manager. CB wrote the manuscript. SD, MFO and NM are investigators of centres, they will carry out recruitment and will collect data. FL is the psychiatrist who 
contributed to the design and coordinates patient care. All authors reviewed and contributed to the manuscript. All authors have read and approved the paper.

Funding The authors have not declared a specific grant for this research from any funding agency in the public, commercial or not-for-profit sectors.

Trial status This is an ongoing trial. Recruitment began in April 2020. We expect to complete recruitment by August 2021 and complete data collection in 0ctober 2021 which is the completion study date. We plan to publish final results in 2021.

Competing interests None declared.

Patient consent for publication Not required.

Provenance and peer review Not commissioned; externally peer reviewed.

Supplemental material This content has been supplied by the author(s). It has not been vetted by BMJ Publishing Group Limited (BMJ) and may not have been peer-reviewed. Any opinions or recommendations discussed are solely those of the author(s) and are not endorsed by BMJ. BMJ disclaims all liability and responsibility arising from any reliance placed on the content. Where the content includes any translated material, BMJ does not warrant the accuracy and reliability of the translations (including but not limited to local regulations, clinical guidelines, terminology, drug names and drug dosages), and is not responsible for any error and/or omissions arising from translation and adaptation or otherwise.

Open access This is an open access article distributed in accordance with the Creative Commons Attribution Non Commercial (CC BY-NC 4.0) license, which permits others to distribute, remix, adapt, build upon this work non-commercially, and license their derivative works on different terms, provided the original work is properly cited, appropriate credit is given, any changes made indicated, and the use is non-commercial. See: http://creativecommons.org/licenses/by-nc/4.0/.

ORCID iD

Charline Bertholdt http://orcid.org/0000-0001-9297-5363

\section{REFERENCES}

1 Dashraath P, Wong JLJ, Lim MXK, et al. Coronavirus disease 2019 (COVID-19) pandemic and pregnancy. Am J Obstet Gynecol 2020;222:521-31.

2 Findeklee S, Morinello E. Clinical implications and economic effects of the corona virus pandemic on gynaecology, obstetrics and reproductive medicine in Germany-learning from Italy. Minerva Ginecol 2020;72:171-7.

3 Kayem G, Lecarpentier E, Deruelle P, et al. A snapshot of the Covid-19 pandemic among pregnant women in France. J Gynecol Obstet Hum Reprod 2020;49:101826.

4 Viaux S, Maurice P, Cohen D, et al. Giving birth under lockdown during the COVID-19 epidemic. J Gynecol Obstet Hum Reprod 2020;49:101785.

5 Bohren MA, Berger BO, Munthe-Kaas H, et al. Perceptions and experiences of labour companionship: a qualitative evidence synthesis. Cochrane Database Syst Rev 2019;3:CD012449.

6 Hahn-Holbrook J, Cornwell-Hinrichs T, Anaya I. Economic and health predictors of national postpartum depression prevalence: a systematic review, meta-analysis, and meta-regression of 291 studies from 56 countries. Front Psychiatry 2017;8:248.

7 Czarnocka J, Slade P. Prevalence and predictors of posttraumatic stress symptoms following childbirth. Br J Clin Psychol 2000;39:35-51.

8 Ayers S, Bond R, Bertullies S, et al. The aetiology of post-traumatic stress following childbirth: a meta-analysis and theoretical framework. Psychol Med 2016;46:1121-34.

9 Boudou M, Séjourné N, Chabrol H. [Childbirth pain, perinatal dissociation and perinatal distress as predictors of posttraumatic stress symptoms]. Gynecol Obstet Fertil 2007;35:1136-42.
10 Montmasson H, Bertrand P, Perrotin F, et al. [Predictors of postpartum post-traumatic stress disorder in primiparous mothers]. $J$ Gynecol Obstet Biol Reprod 2012;41:553-60.

11 Garthus-Niegel S, Horsch A, Handtke E, et al. The impact of postpartum posttraumatic stress and depression symptoms on couples' relationship satisfaction: a population-based prospective study. Front Psychol 2018;9:1728.

12 Bodnar-Deren S, Klipstein K, Fersh M, et al. Suicidal ideation during the postpartum period. J Womens Health 2016;25:1219-24.

13 Orri M, Gunnell D, Richard-Devantoy S, et al. In-Utero and perinatal influences on suicide risk: a systematic review and meta-analysis. Lancet Psychiatry 2019;6:477-92.

14 Shi P, Ren H, Li H, et al. Maternal depression and suicide at immediate prenatal and early postpartum periods and psychosocial risk factors. Psychiatry Res 2018;261:298-306.

15 Berthelot N, Lemieux R, Garon-Bissonnette J, et al. Uptrend in distress and psychiatric symptomatology in pregnant women during the coronavirus disease 2019 pandemic. Acta Obstet Gynecol Scand 2020;99:848-55.

16 Brooks SK, Webster RK, Smith LE, et al. The psychological impact of quarantine and how to reduce it: rapid review of the evidence. The Lancet 2020;395:912-20.

17 Johal SS. Psychosocial impacts of quarantine during disease outbreaks and interventions that may help to relieve strain. $N Z$ Med J 2009;122:47-52.

18 Reynolds DL, Garay JR, Deamond SL, et al. Understanding, compliance and psychological impact of the SARS quarantine experience. Epidemiol Infect 2008;136:997-1007.

19 Zanardo V, Manghina V, Giliberti L, et al. Psychological impact of COVID-19 quarantine measures in northeastern Italy on mothers in the immediate postpartum period. Int J Gynaecol Obstet 2020;150:184-188.

20 Hodnett ED, Simmons-Tropea DA. The labour agentry scale: psychometric properties of an instrument measuring control during childbirth. Res Nurs Health 1987;10:301-10.

21 Cox JL, Holden JM, Sagovsky R. Detection of postnatal depression. development of the 10-item Edinburgh postnatal depression scale. Br J Psychiatry J Ment Sci. juin 1987;150:782-6.

22 Horowitz M, Wilner N, Alvarez W. Impact of event scale: a measure of subjective stress. Psychosom Med 1979;41:209-18.

23 Ware J, Kosinski M, Keller SD. A 12-Item short-form health survey: construction of scales and preliminary tests of reliability and validity. Med Care 1996;34:220-33.

24 Mappa I, Distefano FA, Rizzo G. Effects of coronavirus 19 pandemic on maternal anxiety during pregnancy: a prospectic observational study. J Perinat Med 2020;48:545-50.

25 Biviá-Roig G, La Rosa VL, Gómez-Tébar M, et al. Analysis of the impact of the confinement resulting from COVID-19 on the lifestyle and psychological wellbeing of Spanish pregnant women: an Internet-based cross-sectional survey. Int J Environ Res Public Health 2020;17:17.

26 Wu Y, Zhang C, Liu H, et al. Perinatal depressive and anxiety symptoms of pregnant women during the coronavirus disease 2019 outbreak in China. Am J Obstet Gynecol 2020;223:240. e1-240.e9.

27 Dude A, Fette LM, Reddy UM, et al. Maternal sense of control during childbirth and infant feeding method. Obstetrics \& Gynecology 2020;135:583-90.

28 Geerts CC, Klomp T, Lagro-Janssen ALM, et al. Birth setting, transfer and maternal sense of control: results from the deliver study. BMC Pregnancy Childbirth 2014;14:27.

29 Adewuya AO, Ologun YA, Ibigbami OS. Post-Traumatic stress disorder after childbirth in Nigerian women: prevalence and risk factors. BJOG: Int J O\&G 2006;113:284-8.

30 Durankuș F, Aksu E. Effects of the COVID-19 pandemic on anxiety and depressive symptoms in pregnant women: a preliminary study. $J$ Matern Fetal Neonatal Med 2020;91:1-7. 